\title{
Review
}

\section{Current status of IMRT in head and neck cancer}

\author{
Jaime Gomez-Millan ${ }^{c, *}$, Jesús Romero Fernández ${ }^{b}$, \\ Jose Antonio Medina Carmona ${ }^{a}$ \\ a Department of Radiation Oncology, Hospital Virgen de la Victoria, Campus Teatinos s/n, 29010 Málaga, Spain \\ b Department of Radiation Oncology, Hospital Puerta de Hierro, Calle de Manuel de Falla, 1, 28222 Majadahonda, \\ Madrid, Spain \\ c Servicio de Oncologia Radioterapica del Hospital Clínico Virgen de la Victoria, Campus Teatinos s/n, 29010 Málaga, \\ Spain
}

\section{A R T I C L E I N F O}

Article history:

Received 30 April 2013

Received in revised form

24 August 2013

Accepted 23 September 2013

\section{Keywords:}

Intensity modulated radiation

therapy

Head and neck cancer

Rotational therapy

Tomotherapy

\begin{abstract}
A B S T R A C T
Background: IMRT provides highly conformal dose distributions creating non uniform spatial intensity using different segments in the beam.

Material \& Methods and Results: Different retrospective studies have shown a high capability of IMRT to treat tumours close to the base of skull. Prospective studies have shown a decrease in xerostomia compared with conventional 3D conformal treatment (3DCRT). Modulation of intensity is performed by the movement of the multileaf collimator (MLC) that can deliver the radiation in different ways, such as static field segments, dynamic field segments and rotational delivery (arc therapy and tomotherapy). There are slight differences among the different techniques in terms of homogeneity, dose conformity and treatment delivery time. Conclusions: The best method to deliver IMRT will depend on multiple factors such as deliverability, practicality, user training and plan quality.
\end{abstract}

() 2013 Greater Poland Cancer Centre. Published by Elsevier Urban \& Partner Sp. z o.o. All

rights reserved.

\section{Background}

Radiotherapy (RT) is the cornerstone of treatment for locally advanced head and neck cancer (LAHNC). The main goal of radiotherapy is to provide the maximum loco-regional control with the minimum toxicity. However, the complex relationship between tumours and critical structures, with concave shapes and very close interrelation, limits the ability of conventional radiotherapy to shape the doses to the target volumes and to spare the organs at risk (OAR). In intensity modulated radiation therapy (IMRT), modulation of the beam fluence permits to deliver a non-uniform intensity to the target, ${ }^{1}$ increasing the conformation of the high dose to the tumour. The aim of this revision is to review the basis of IMRT and different methods to deliver this technology.

\section{Concept of IMRT and differences with 3DCRT}

The transition from conventional 2D treatment planning to 3D conformal treatment (3DCRT) has been an important advance in radiation technology. In 3DCRT, simulation and planning are performed based on computed tomography images, achieving a precise tumour definition and a more accurate dose

\footnotetext{
* Corresponding author. Tel.: +34 951032686; fax: +34 952279407.

E-mail addresses: jaimegomezmillan@gmail.com (J. Gomez-Millan), jesus.romero@salud.madrid.org (J.R. Fernández), jmedinacarmona@gmail.com (J.A. Medina Carmona). 
calculation by accounting for axial anatomy and complex tissue contours. Moreover, it permits to use multiple fields, including oblique and non-coplanar, which together with variations in weight, wedges and shaped blocks or multileaf collimators (MLC) permits to achieve adequate tumour coverage and normal tissue sparing.

One of the main differences between IMRT and 3DCRT is the ability to conform the dose distribution to the target. In most of the techniques to deliver IMRT, MLC divides beam fields in different segments, or creates "segments" moving across the field, getting a different fluence in each beam. Modulation of the fluence creates non-uniform spatial intensity distributions that produce highly conformal dose distributions.

\section{Steps involved in IMRT}

Three of the most crucial parts of IMRT are target delineation, treatment planning and quality assurance. Because of the resulting steeper absorbed-dose gradients, optimal IMRT requires more accurate delineation of both tumour and normal tissue than does conventional radiotherapy. However, there is a substantial heterogeneity in target definition and prescription among radiation oncologists with IMRT expertise that will make it difficult to assess the success of the treatment. Efforts to standardize and simplify the IMRT process have been suggested for Head and Neck IMRT practice. ${ }^{2}$ Moreover, additional normal tissue often has to be delineated because structures that are not specified are not considered in the planning process, and may receive a significant high absorbed dose. Optimization is a key point in radiotherapy planning. Using MLC, it is possible to create a wide range of beam intensities employing different segments. Optimization explores these possibilities to find the optimum intensity pattern for the desired outcome, that is specified with dose and volume constraints. The planning process can be summarized in three points: first, the desired outcome is specified in terms of dose and volume constraints and objectives for OAR and PTV using a system of priorities. Second, an objective function will be constructed to specify the goodness of the plan. During the optimization process, a candidate fluence distribution map with a cost function as close as possible to the objective function is searched. Finally, once the optimal fluence map is found, it must be converted into deliverable field segments according to the specified method of delivery, considering the limitations of the treatment unit due to physical and mechanical characteristics of the MLC. Dose calculation algorithms based in beamlet optimization, such as convolution/superposition (each field is discretized into a grid of beamlets with distinct intensity), or aperture-based optimization (the best set of aperture shapes is found to deliver the intensity pattern without discretization of the field in beamlets), will provide accurate absorbed-dose calculations. ${ }^{3}$

\section{Image-guided radiotherapy (IGRT)}

Delivery of a high radiation dose to the clinical target volume avoiding critical structures increases the complexity of treatment planning and delivery and also the precision required for localization and for securing geometrical precision. IMRT has an excellent capability to put the dose where it is needed on a screen. However, it is necessary to have in mind that planning CT is a snap shot and may not represent the every day location. The advent of on board cone beam computed tomography (CBT) and in room CT with high soft tissue contrast has opened new opportunities for higher accuracy in radiotherapy. ${ }^{4}$ Other image-guidance capable systems that have an important role in IGRT are ultrasonography, MRI, and optical imaging techniques. ${ }^{5}$ Cone beam may be performed before every fraction and corrections are made on line after aligning with the planning CT, reducing both the systematic and random position. ${ }^{6}$ Thus, the information derived could be used to evaluate more accurately the PTV margin required for IMRT. Validated protocols of systematic error correction can minimize the IGRT workload. ${ }^{7}$

Although supplementary imaging involved in IGRT exposes the patient to more radiation, with an extra dose of $0.1-3 \%$ of the treatment total dose, this technique guarantees a precise administration of radiation at the right place. Moreover, a reduction in the PTV margin will decrease the dose to critical structures. $^{8}$

Patients with head and neck cancer may experience significant changes in the tumour volume and anatomical structures during the radiotherapy course. This issue has a special significance considering that IMRT achieves highly conformal dose distributions. It has been shown that the tumour volume can dramatically decrease during a treatment course, and that replanning considering this volume change would translate into a substantial sparing of the surrounding critical structures. ${ }^{9}$ Moreover, patients treated also with chemotherapy may loose considerable weight during their full radiation treatment, and dose in critical structures may change as a result of this weight loss. ${ }^{10}$ Image guidance can allow to visualize the anatomical changes during the treatment, and if dose distribution at some point reveals that there are low dose regions or a higher dose region in a critical structure, subsequent fractions could be re-planned.

\section{IMRT: clinical or dosimetric benefit?}

Different retrospective studies have shown the increased therapeutic ratio achievable with IMRT in tumours close to the base of the skull, such as paranasal sinus cancer. ${ }^{11}$ One of the most frequent chronic and invalidating side effects in LAHNC is xerostomia, with a well documented association with mean doses to the parotid gland of more than $26 \mathrm{~Gy} .{ }^{12}$ Moreover, it has been recently shown that with parotid doses lower than 25-30 Gy function recovery is substantial and a return to pre-treatment levels may be achieved in 2 years after RT. ${ }^{13}$ A recent randomized study with 94 patients with locally advanced pharynx cancer has shown that IMRT resulted in a decrease of xerostomia (Grade $\geq 2$ ) at 12 and 24 months compared with 3DCRT, with an absolute benefit of $54 \%$ at 24 months. ${ }^{14}$ IMRT allows to achieve heterogeneous dose distributions and can be exploited to treat simultaneously elective and primary volumes decreasing the overall time of the treatment, with a potential 
benefit derived from reducing the impact of accelerated proliferation. However, for efficacy endpoints, there are no robust data to advocate IMRT. ${ }^{15,16}$ High quality studies are needed to determine the comparative effectiveness of IMRT.

There are potential disadvantages of IMRT that should be considered. As a result of leakage and scattered radiation, an IMRT plan results in an increase in absorbed dose outside the treated volume. ${ }^{17}$ It has been estimated that the incidence of secondary malignancies may increase from $1 \%$ to $1.75 \%,{ }^{18}$ and it raises special concerns in paediatric patients. ${ }^{19}$ However studies are needed to determine the incidence of second tumours compared with other techniques. Some authors have claimed that recurrences may present in regions that would have been previously irradiated with 3DCRT technique. However, different studies have shown that despite the high conformality of dose distributions, geographical misses are uncommon. ${ }^{20}$ Finally, because the resulting steep dose gradient, IMRT involves more accurate delineation than conventional radiotherapy, and tissues that may receive unexpected high doses have to be contoured. Moreover, IMRT has a more stringent machine quality assurance (QA) to check the performance of the IMRT delivery system. ${ }^{21}$

IMRT requires greater time and resource commitment than conventional radiotherapy. Intensity modulated radiation therapy employs automated iterative optimization techniques that take more time than 3D-CRT treatment planning. Moreover, IMRT has more stringent machine quality assurance to check the performance of the IMRT delivery system.

\section{Delivery of IMRT}

Modulation of intensity may be performed by MLC, a large number of highly absorbing tungsten leaves that can be moved against each other to create a large variety of field openings. ${ }^{22}$ The movement of MLC under a computer control can be used to produce various forms of intensity modulation. Modulation can be achieved by three main ways: IMRT with static field segments, IMRT with dynamic delivery, and IMRT rotational therapy. In IMRT with static field segments (step and shoot), the field is divided in different segments with uniform beam levels, and radiation is not delivered while the leaves move to create the next segment. ${ }^{23}$ In dynamic IMRT the radiation is delivered as the leaves are moving. The leaves of MLC move across the field, and the time-dependent position of each leaf determines the intensity. ${ }^{24}$ Finally, rotational therapy can be delivered using intensity modulated arc therapy (IMAT) or tomotherapy. In the IMAT, MLC has the leaf pattern changing continuously as the gantry rotates, allowing the simultaneous variation in dose rate. ${ }^{25}$ More than one rotational arch with different patterns of radiation can be required to get the optimal distribution. ${ }^{25}$

Several investigations have compared dosimetric results of IMAT versus IMRT with static fields. Most of the published data compare the different techniques in terms of target volume coverage, conformity index (the degree to which the high dose region conforms to the target volume), homogeneity index (uniformity of absorbed-dose distribution), monitor units and time required to deliver the radiation. Results suggest that both techniques are almost equivalent in volume coverage, dose conformity and dose homogeneity, with a slight reduction with IMAT (VMAT, Elekta) in dose to OAR. Lee et al. ${ }^{26}$ compared the planning performance of IMAT versus sequential IMRT for nasopharyngeal carcinoma, finding a similar target coverage, with a $2 \%$ benefit in sparing OAR with IMAT (VMAT, Elekta). The major difference was the improved efficiency of IMAT, resulting in a faster delivery time and the use of fewer MU/fr. Stieler et al. compared IMRT with 7 or 9 static fields and IMAT (VMAT, Elekta), showing all plans to be of an excellent quality in homogeneity, target coverage and conformity. Again, the major difference was the higher efficiency of VMAT, with faster delivery time $(7.6,8.5$ and 4.3 , respectively) and the use of fewer MU/fr. ${ }^{27}$ Other investigations have confirmed these findings. ${ }^{28,29}$ It has to be considered that results of comparisons between different techniques are performed with specific conditions of linear accelerators, number of beams and segments, and should not be generalized to other treatment conditions.

\section{Tomotherapy}

Helical tomotherapy $(\mathrm{HT})$ is a combination of a compact $6 \mathrm{MV}$ linear accelerator, and a megavoltage computed tomography (MVCT) detection system. A binary multileaf collimator consisting of 64 leaves of $6.5 \mathrm{~mm}$, which can be "open" or "closed", generates multiple shaped beams called "beamlets". The length of time a leaf is in the open position determines the intensity delivered by the "beamlet". The dose is delivered in a helical manner: the gantry continuously rotates while the patient couch is translated in the rotation plane. The MVCT, as other IGRT systems, can be used for patient setup and repositioning; visualization of tumoral or anatomical changes during treatment, and reconstruction of the actual dose received by the patient. Although MVCT images have a lower contrast than KV-CT, their quality are sufficient for setup verification and tumour identification. Although the optimal frequency of image registration has not been defined, patient setup can be improved if daily imaging is performed. ${ }^{30}$

The major components of the HT process comprise 3D imaging generated by conventional CT; definitions of target volumes and organs at risk (OARs); optimized HT planning; creation of verification data and phantom irradiation; transfer of planning data to the treatment unit; pre-treatment MVCT; corrections of patient setup if needed; treatment delivery; and, optionally, dose reconstruction.

In head and neck cancer patients, mean treatment times are longer for HT than in other modalities. In a report of Kruser et al., the number of MU, varied significantly between the 3DCRT (median= 502), step-and-shoot IMRT (median=1087), and HT (median $=6757$ ) $\cdot{ }^{31}$ Moreover, Head and Neck HT treatment times have been reported in the range of 4.8-7.74 min; longer than for Rapid-Arc, 1.4-3.05 min, and VMAT, 4.6 min. ${ }^{32}$

Several studies have reported the dosimetric characteristics of tomotherapy and compared them with other IMRT modalities. Helical tomotherapy may produce better homogeneous doses as compared with other IMRT systems. A dosimetric study demonstrate that both IMAT (VMAT, Elekta) 
and HT are capable of providing more uniform target doses and improved normal tissue sparing as compared with fixed field IMRT. ${ }^{33}$ In a comparison of HT with step-and-shoot IMRT for nasopharyngeal carcinoma, a significant gain was observed in the conformation index (CI) and homogeneity index (HI) of $11.9 \%$ and $8.8 \%$, respectively, favouring $\mathrm{HT}^{34}{ }^{34}$ In others studies the conformity and homogeneity within the PTV were improved for HT over IMAT (VMAT, Elekta) and IMRT. $^{35}$

Due to its dose conformation capabilities, HT diminished the dose received by the OARs, mainly by the parotid glands. HT have significantly better sparing of salivary glands than segmental multileaf collimator (SMLC)-IMRT (mean dose of 27.3 vs. $34.1 \mathrm{~Gy}, p=0.03) .{ }^{36}$ Another study confirmed a lower dose to the parotids for HT as compared with IMAT (VMAT, Elekta, and RapidArc, Varian Medical Systems) with doses of $14.1 \mathrm{~Gy}, 23 \mathrm{~Gy}$ and $26.5 \mathrm{~Gy}$, respectively. ${ }^{37}$ In nasopharyngeal carcinoma, compared to step-and-shoot IMRT, HT reduced the dose not only to parotids but also to brainstem $(p=0.02)$, larynx $(p=0.03)$, and oral cavity $(p=0.03) .{ }^{38}$ Nguyen et al. ${ }^{39}$ reported a mean total cochlea dose of 36 Gy for IMRT compared to $12.1 \mathrm{~Gy}$ for HT $(p=0.002)$ in patients with LAHNC. In a dosimetric analysis, HT reduced the maximum doses to the optic chiasm, as well as to the ipsilateral optic nerve and retina as compared with step-and-shoot IMRT $(P<0.05) .{ }^{40}$

There are a scarce number of non-randomized studies reporting outcomes and normal tissue complications with HT. The overall survival and loco-regional control for HT are similar to that reported in the literature for other IMRT techniques for oropharyngeal carcinoma ${ }^{41}$; sinonasal carcinoma $42^{42}$; oral cavity ${ }^{43}$ and nasopharynx. ${ }^{34}$ Recently, a retrospective study with a small number of patients has founded an increase in recurrence free survival with $\mathrm{HT}$ compared to conventional radiotherapy. ${ }^{44}$

\section{Conclusions}

IMRT creates non-uniform spatial intensity distributions to achieve highly conformal dose distributions, decreasing doses to OAR. However, no increase in survival or control has been shown. There are different methods to deliver IMRT, with similar advantages in conformality and homogeneity. The main advantage of IMAT is the shorter time to deliver a fraction of radiation. Some studies have shown an advantage of HT in conformality and homogeneity, with lower doses to organs at risk. On the other hand, a longer treatment time has also been described. The best method to deliver IMRT depends on multiple factors, such as plan quality, deliverability, practicality, user training, individual cases.

\section{Conflict of interest}

All authors have no conflicts of interest.

\section{Financial disclosure}

None declared.

\section{REFEREN CES}

1. Borfeld T. IMRT: a review and preview. Phys Med Biol 2006;51:363-79.

2. Hong TS, Tome WA, Harari PM. Radiother Oncol 2012;103(1):92-8.

3. ICRU83. ICRU report 83 (2010): prescribing, recording, and reporting photon-beam intensity-modulated radiation therapy (IMRT); 2010.

4. Jaffray D. Emergent technologies for 3-dimensional image-guided radiation delivery. Semin Radiat Oncol 2005;153:208-16.

5. Simpson DR, Lawson JD, Nath SK. A survey of image-guided radiation therapy use in the United States. Cancer 2010;116:3953-60.

6. Forrest LJ, Mackie TR, Ruchala K. The utility of mega-voltage computed tomography images from a helical tomotherapy system for setup verification purposes. Int J Radiat Oncol Biol Phys 2004;60:1639-44.

7. Bujold A, Craig T, Jaffray D. Image-guided radiotherapy. Has it influenced patient outcomes? Semin Radiat Oncol 2012;22:50-61.

8. Ding GX, Coffey C. Radiation dose from kilovoltage cone beam computed tomography in an image-guided radiotherapy procedure. Int J Radiat Oncol Biol Phys 2009;73(2):610-7.

9. Geets X, Tomsej M, Lee JA. Adaptive biological image-guided IMRT with anatomic and functional imaging in pharyngo-laryngeal tumors: impact on target volume delineation and dose distribution using helical tomotherapy. Radiother Oncol 2007;85:105-15.

10. Lee C, Langen KM, Lu W. Assessment of parotid gland dose changes during head and neck cancer radiotherapy using daily megavoltage computed tomography and deformable image registration. Int J Radiat Oncol Biol Phys 2008;71(5):1563-71.

11. Claus F, Minheer B, Rasch C. Report of a study on IMRT planning strategies for ethmoid sinus cancer. Strahlenther Onkol 2002;178(10):572-6.

12. Eisbruch A, Ship JA, Martel MK, et al. Parotid gland sparing in patients undergoing bilateral head and neck irradiation: techniques and early results. Int J Radiat Oncol Biol Phys 1996;36:469-80.

13. Li Y, Taylor JM, Ten Haken RK, et al. The impact of dose on parotid salivary recovery in head and neck cancer patients treated with radiation therapy. Int J Radiat Oncol Biol Phys 2007;67:660-9.

14. Nutting CM, Morden JP, Harrington KJ, et al. PARSPORT trial management group: parotid-sparing intensity modulated versus conventional radiotherapy in head and neck cancer (PARSPORT): a phase 3 multicentre randomised controlled trial. Lancet Oncol 2011;12:127-36.

15. De Neve W, De Gersem W, Madani I. Rational use of intensity-modulated radiation therapy: the importance of clinical outcome. Semin Radiat Oncol 2012;22(1):40-9.

16. O'ullivan B, Rumble BB, Warde P. Intensity-modulated radiotherapy in the treatment of head and neck cancer. Clin Oncol (R Coll Radiol) 2012;24(7):474-87.

17. Williams PC, Hounsell AR. X-ray linkage considerations for IMRT. Br J Radiol 2001;74:98-100.

18. Hall EJ, Wuu CS. Radiation induced second cancers: the impact of 3D-CRT and IMRT. Int J Radiat Oncol Biol Phys 2003;56:83-8.

19. Mansur EE, Klein BP, Maserang. Measured peripheral dose in pediatric radiation therapy: a comparison of intensity-modulated and conformal techniques. Radiother Oncol 2007;822:179-87. 
20. Gregoire V, De Neve W, Eisbruch A. Intensity-modulated radiation therapy for head and neck carcinoma. Oncologist 2007;12(5):555-64.

21. Klein EE, Hanley J, Bayouth J. Task group 142 report: quality assurance of medical accelerators. Med Phys 2009;36:4197-212.

22. Galvin JM, Smith AR, Lally B. Characterization of a multi-leaf collimator system. Int J Radiat Oncol Biol Phys 1993;25:181-92.

23. Bortfeld TR, Kahler DL, Waldron TJ, Boyer AL. X-ray field compensation with multileaf collimators. Int J Radiat Oncol Biol Phys 1994;28:723-30.

24. Dirkx MLP, Heijmen BJM, Santvoort JPC. Leaf trajectory calculation for dynamic multi-leaf collimation to realize optimized fluence profiles. Phys Med Biol 1998;43:1171-84.

25. Yu CX. Intensity-modulated arc therapy with dynamic multileaf collimation: an alternative to tomotherapy. Phys Med Biol 1995;40:1435-49.

26. Lee TF, Chao PJ, Ting HM. Comparative analysis of SmartArc-based dual arc volumetric-modulated arc radiotherapy (VMAT) versus intensity modulated radiotherapy (IMRT) for nasopharyngeal carcinoma. J Appl Clin Med Phys 2011;12(4):3587.

27. Stieler F, Wolff D, Schmid H. A comparison of several modulated radioteraphy techniques for head and neck cancer and dosimetric validation of VMAT. Radiother Oncol 2011;101(3):388-93.

28. Bertelsen A, Hansen CR, Johansen J, et al. Single arc volumetric modulated arc therapy of head and neck cancer. Radiother Oncol 2010;95:142-8.

29. Guckenberger M, Richter A, Krieger T, et al. Is a single arc sufficient in volumetric-modulated arc therapy (VMAT) for complex-shaped target volumes? Radiother Oncol 2009;93:259-65.

30. Duma MN, Kampfer S, Schuster T, et al. Do we need daily image-guided radiotherapy by megavoltage computed tomography in head and neck helical tomotherapy? The actual delivered dose to the spinal cord. Int J Radiat Oncol Biol Phys 2012;84:283-8.

31. Kruser TJ, Rice SR, Cleary KP, et al. Acute hematologic and mucosal toxicities in head and neck cancer patients undergoing chemoradiotherapy: a comparison of 3D-CRT IMRT, and helical tomotherapy. Technol Cancer Res Treat 2013 [Epub ahead of print].

32. Van Gestel D, van Vliet-Vroegindeweij C, Van den Heuvel F, et al. RapidArc, SmartArc and TomoHD compared with classical step and shoot and sliding window intensity modulated radiotherapy in an oropharyngeal cancer treatment plan comparison. Radiat Oncol 2013;8:37.

33. Rao M, Yang W, Chen F, et al. Comparison of Elekta VMAT with helical tomotherapy and fixed field IMRT: plan quality, delivery efficiency and accuracy. Med Phys 2010;37: 1350-9.

34. Lee TF, Fang FM, Chao P, et al. Dosimetric comparisons of helical tomotherapy and step-and-shoot intensity-modulated radiotherapy in nasopharyngeal carcinoma. Radiother Oncol 2008;89:89-96.

35. Clemente S, Wu B, Sanguineti G, et al. SmartArc-based volumetric modulated arc therapy for oropharyngeal cancer: a dosimetric comparison with both intensity-modulated radiation therapy and helical tomotherapy. Int J Radiat Oncol Biol Phys 2011;80:1248-55.

36. Chen AM, Yang CC, Marsano J, et al. Intensity-modulated radiotherapy for nasopharyngeal carcinoma: improvement of the therapeutic ratio with helical tomotherapy vs segmental multileaf collimator-based techniques. Br J Radiol 2012;85:537-43.

37. Wiezorek T, Brachwitz T, Georg D, et al. Rotational IMRT techniques compared to fixed gantry IMRT and Tomotherapy: multi-institutional planning study for head-and-neck cases. Radiat Oncol 2011;6:20.

38. Chen A, Lee N, Yang C, et al. Comparison of intensity-modulated radiotherapy using helical tomotherapy and segmental multileaf collimator-based techniques for nasopharyngeal carcinoma: dosimetric analysis incorporating quality assurance guidelines from RTOG 0225. Technol Cancer Res Treat 2010;9:291-8.

39. Nguyen NP, Vos P, Vinh-Hung V, et al. Feasibility of image-guided radiotherapy based on helical tomotherapy to reduce contralateral parotid dose in head and neck cancer. BMC Cancer 2012;12:175.

40. Chen AM, Sreeraman R, Mathai M, et al. Potential of helical tomotherapy to reduce dose to the ocular structures for patients treated for unresectable sinonasal cancer. Am J Clin Oncol 2010;33:595-8.

41. Shueng PW, Wu LJ, Chen SY, et al. Concurrent chemoradiotherapy with helical tomotherapy for oropharyngeal cancer: a preliminary result. Int J Radiat Oncol Biol Phys 2010;77:715-21.

42. Duthoy W, Boterberg T, Claus F, et al. Postoperative intensity-modulated radiotherapy in sinonasal carcinoma. Clinical results in 39 patients. Cancer 2005;104: 71-82.

43. Hsieh CH, Kuo YS, Liao LJ, et al. Image-guided intensity modulated radiotherapy with helical tomotherapy for postoperative treatment of high-risk oral cavity cancer. BMC Cancer 2011;11:37.

44. Kong M, Hong Se, Choi J. Comparison of survival rates between patients treated with conventional radiotherapy and helical tomotherapy for head and neck cancer. Radiat Oncol 2013;31(1):1-11. 